\title{
Perceptions of "Urgency" in Women With Interstitial Cystitis/Bladder Pain Syndrome or Overactive Bladder
}

\author{
J. Quentin Clemens, ${ }^{1 *}$ Laura M. Bogart, ${ }^{2}$ Karin Liu, ${ }^{3}$ Chau Pham, ${ }^{3}$ Marika Suttorp, ${ }^{3}$ and Sandra H. Berry ${ }^{3}$ \\ ${ }^{1}$ University of Michigan Medical Center, Ann Arbor, Michigan \\ ${ }^{2}$ Harvard University, Boston, Massachusetts \\ ${ }^{3}$ RAND Corporation, Santa Monica, California
}

Purpose: To compare urgency symptoms in women with interstitial cystitis/bladder pain syndrome (IC/BPS) and overactive bladder (OAB). Materials and Methods: Women with diagnoses of IC/BPS $(n=194)$ and OAB $(n=85)$ were recruited from the clinical practices of Urologists $(n=8)$ and Gynecologists $(n=16)$ with recognized expertise in the diagnosis and management of these conditions. Subjects completed a comprehensive telephone survey about their current symptoms. The questionnaire included 11 questions about urinary urgency. Responses were compared between the two groups. Results: Urgency was commonly reported as a symptom by women with both conditions (81\% IC/BPS and $91 \%$ OAB). Compared with IC/BPS, urgency in OAB more often resulted in leakage, and was perceived to be more of a problem. In IC/BPS, the urgency was primarily reported as due to pain, pressure, or discomfort, while in OAB the urgency was more commonly due to fear of leakage. However, approximately $40 \%$ of women with OAB also report urgency due to pain, pressure, or discomfort. Similar proportions of both groups ( $60 \%)$ indicated that the urgency occurred "suddenly" instead of more gradually over a period of minutes or hours. Conclusions: Urgency symptoms differed in women diagnosed with IC/BPS versus those diagnosed with OAB, but there was significant overlap. This suggests that "urgency" is not a well-defined and commonly understood symptom that can be utilized to clearly discriminate between IC/BPS and $\mathrm{OAB}$. These findings reinforce the clinical observation that it is often challenging to differentiate between these two conditions. Neurourol. Urodynam. 30:402-405, 2011. 둔 2010 Wiley-Liss, Inc.

Key words: sensations; specificity; symptoms

\section{INTRODUCTION}

Urologists and Urogynecologists frequently encounter patients who complain of bothersome bladder symptoms such as frequency, nocturia, urgency, or pelvic discomfort, in the absence of an identifiable etiology. Based on the nature of these symptoms, their course over time, and their responsiveness to treatment, clinicians may assign a specific clinical diagnosis. Two commonly assigned diagnoses are overactive bladder (OAB), and interstitial cystitis/bladder pain syndrome (IC/BPS). Considerable effort has been spent in developing definitions which can help clinicians to discriminate between these two conditions. $^{1,2}$

One area of confusion has been related to the symptom of "urgency." The terms "urge" and "urgency" have been used to define symptoms that are characteristic of both IC/BPS and $\mathrm{OAB}, 1,3$ and urgency is included as a descriptor in symptom indices for both $\mathrm{BPS}^{4-6}$ and $\mathrm{OAB} .^{7}$ Some investigators have indicated that the urgency in $\mathrm{OAB}$ is due to a fear of incontinence, while the urgency (or urge to void) in IC/BPS is due to pain. ${ }^{2}$ It has also been suggested that the definition of urgency includes the idea that it is experienced as "sudden," and that "sudden" urgency is less common in IC/BPS than in OAB. ${ }^{2,8}$ However, to date the detailed characteristics of urgency have not been directly compared in cohorts of patients diagnosed with OAB and IC/BPS.

\section{MATERIALS AND METHODS}

The RAND Interstitial Cystitis Epidemiology (RICE) protocol has been previously described in detail. ${ }^{9}$ In the institutional review board approved study, we contacted Urologists and Gynecologists with recognized expertise in the management of IC/BPS, OAB, and other pelvic conditions, and invited them to refer female patients with these conditions to the RICE investigative team. These physicians included academicians and community physicians from across the United States. Eight Urologists and 16 Gynecologists participated, and a total of 194 subjects with IC/BPS and 85 with OAB were recruited. Additional women who had been diagnosed with both conditions were excluded from this analysis. Study subjects completed a comprehensive 90-minute telephone questionnaire conducted by trained interviewers at RAND. Eleven questions about urinary urgency were included in the questionnaire. Responses to these urgency questions were compared between the IC/BPS and OAB groups using the chi-squared test for proportions.

\section{RESULTS}

Mean age of the women with IC/BPS was 47.5 (range 18-85), and mean age of the women with OAB was 62.3 (range 21-85;

Conflicts of interest: Dirk De Ridder led the editorial process.

Dr. Clemens:Merck - equity interest Pfizer - consultant, trial participation Medtronic - consultant Lilly - consultant Afferent Pharmaceuticals, Inc - consultant UpToDate - royalties.

*Correspondence to: J. Ouentin Clemens M.D., MSCI Department of Urology, University of Michigan Medical Center, 1500 East Medical Center Drive, Taubman Center 3875, Ann Arbor, MI 48109-5330.E-mail: qclemens@umich.edi Received 13 April 2010; Accepted 9 June 2010

Published online 06 November 2010 in Wiley Online Library (wileyonlinelibrary.com).

DOI 10.1002/nau.20974 
TABLE I. Questionnaire Responses in Women with IC/BPS and OAB

\begin{tabular}{|c|c|c|c|c|}
\hline Question & Responses & IC/PBS (\%) & $\mathrm{OAB}(\%)$ & $P$-value \\
\hline \multirow{2}{*}{$\begin{array}{l}\text { During the past } 3 \text { months have you had a strong feeling that you had to urinate that } \\
\text { made it difficult for you to wait to go to the bathroom? }\end{array}$} & Yes & 80.5 & 91.2 & 0.008 \\
\hline & No & 19.5 & 8.8 & \\
\hline \multirow{3}{*}{$\begin{array}{l}\text { Once this feeling of urgency starts, do you leak urine right away without being able } \\
\text { to control it, or do you leak urine only if you have to wait for a while before you can } \\
\text { go to the bathroom? }\end{array}$} & Leak right away & 13.4 & 56.3 & $<0.001$ \\
\hline & Leak if have to wait & 49.0 & 33.0 & \\
\hline & Don't leak & 37.6 & 10.7 & \\
\hline \multirow{4}{*}{$\begin{array}{l}\text { Would you say this urge to urinate is mainly because of pain, pressure, or discomfort } \\
\text { or would you say this urge to urinate is mainly because you are afraid you will not } \\
\text { make it to the bathroom in time to avoid wetting? }\end{array}$} & Pain, pressure, discomfort & 86.9 & 41.8 & $<0.001$ \\
\hline & Afraid of leakage & 11.0 & 48.5 & \\
\hline & Both & 0.7 & 3.9 & \\
\hline & Neither & 1.4 & 5.8 & \\
\hline \multirow{5}{*}{$\begin{array}{l}\text { During the past month, how much has the need to urinate with little warning been a } \\
\text { problem for you? }\end{array}$} & No problem & 23.4 & 11.5 & 0.01 \\
\hline & Very small problem & 17.0 & 10.7 & \\
\hline & Small problem & 15.3 & 23.0 & \\
\hline & Medium problem & 24.7 & 27.9 & \\
\hline & Big problem & 19.6 & 27.1 & \\
\hline \multirow{6}{*}{$\begin{array}{l}\text { During the last month, how often have you had a strong urge or pressure to urinate } \\
\text { immediately, with no, or little warning? }\end{array}$} & Do not have the symptom & 18.2 & 8.0 & 0.01 \\
\hline & Rarely & 23.1 & 17.6 & \\
\hline & A few times & 18.2 & 28.8 & \\
\hline & Fairly often & 16.1 & 20.8 & \\
\hline & Usually & 10.6 & 6.4 & \\
\hline & Almost always & 13.6 & 18.4 & \\
\hline \multirow{3}{*}{$\begin{array}{l}\text { Does this urge or feeling that you need to urinate usually start suddenly and without } \\
\text { any warning or do you usually feel it coming on over a period of time that could be } \\
\text { minutes or hours? }\end{array}$} & Suddenly & 56.3 & 61.5 & 0.673 \\
\hline & Over time & 40.9 & 35.6 & \\
\hline & Don't know & 2.8 & 2.9 & \\
\hline \multirow{6}{*}{$\begin{array}{l}\text { During the past month, how often have you felt the strong need to urinate with little } \\
\text { or no warning? }\end{array}$} & Not at all & 18.9 & 12.2 & 0.30 \\
\hline & $<1$ time in 5 & 15.9 & 13.0 & \\
\hline & $<$ Half the time & 16.3 & 17.9 & \\
\hline & About half the time & 17.2 & 19.5 & \\
\hline & $>$ Half the time & 15.5 & 13.0 & \\
\hline & Almost always & 16.3 & 24.4 & \\
\hline \multirow[t]{6}{*}{ During the last month, how often have you had difficulty postponing urination? } & Do not have the symptom & 14.1 & 8.8 & 0.21 \\
\hline & Rarely & 18.8 & 14.4 & \\
\hline & A few times & 18.0 & 24.8 & \\
\hline & Fairly often & 20.1 & 21.6 & \\
\hline & Usually & 12.0 & 8.0 & \\
\hline & Almost always & 17.1 & 22.4 & \\
\hline \multirow{6}{*}{$\begin{array}{l}\text { In the last } 7 \text { days, how many times did you feel a strong urge or pressure that signaled } \\
\text { the need to urinate immediately, whether or not you urinated or leaked urine? }\end{array}$} & Not at all & 19.2 & 14.4 & 0.79 \\
\hline & Once & 8.9 & 12.0 & \\
\hline & A few times $(2-3)$ & 22.6 & 25.6 & \\
\hline & Several times (4-6) & 10.2 & 9.6 & \\
\hline & Many times $(7+)^{\prime}$ & 10.6 & 12.0 & \\
\hline & Every day & 28.5 & 26.4 & \\
\hline \multirow{6}{*}{$\begin{array}{l}\text { During the last month or so, how often have you found it difficult to postpone } \\
\text { urination? }\end{array}$} & Do not have the symptom & 11.9 & 10.4 & 0.46 \\
\hline & $<1$ time in 5 & 14.4 & 13.6 & \\
\hline & $<$ Half the time & 18.2 & 14.4 & \\
\hline & About half the time & 15.3 & 15.2 & \\
\hline & $>$ Half the time & 17.4 & 13.6 & \\
\hline & Almost always & 22.9 & 32.8 & \\
\hline \multirow{5}{*}{$\begin{array}{l}\text { How much of the time during the past } 3 \text { months have you had the feeling of a strong } \\
\text { urge or feeling that you had to urinate that made it difficult to wait to go to the } \\
\text { bathroom? }\end{array}$} & Occasionally & 17.9 & 18.4 & 0.84 \\
\hline & Some of the time & 17.9 & 16.7 & \\
\hline & Half of the time & 24.2 & 20.2 & \\
\hline & Most of the time & 26.8 & 32.5 & \\
\hline & All of the time & 13.2 & 12.3 & \\
\hline
\end{tabular}

$P<0.001)$. Questionnaire responses for the two groups are provided in the Table I. Responses to 5 of the 11 urgency questions were statistically different between the two patient groups, although there was considerable overlap in how the symptom of urgency was experienced. During the preceding 3 months, "a strong feeling to urinate that made it difficult to wait to go to the bathroom" was reported by most patients, but by more OAB patients $(91 \%)$ than IC/BPS patients $(81 \%$; $P<0.008)$. As expected, more $\mathrm{OAB}$ patients reported urgency associated with urinary incontinence; only $11 \%$ of $O A B$ patients reported no urinary incontinence related to urgency, compared with $38 \%$ of IC/BPS patients. Conversely, $56 \%$ of OAB patients reported they leak urine "right away" when the feeling of urgency starts, com- pared with $13 \%$ of IC/BPS patients. The nature of the urgency was also different between the two groups. In IC/BPS patients, fully $87 \%$ reported that the urgency was due to pain, pressure, or discomfort, while only $11 \%$ indicated the urgency was due to a fear of incontinence. By comparison, $42 \%$ of OAB patients reported that the urgency was due to pain, pressure, or discomfort, while $49 \%$ indicated the urgency was due to fear of incontinence. The need to urinate with little warning appeared to be more bothersome for OAB patients, as this symptom was reported to be "no problem" or a "very small problem" by $40 \%$ of IC/BPS patients compared with $22 \%$ of OAB patients. This same symptom was a "big problem" for $27 \%$ of OAB patients and $20 \%$ of IC/BPS patients. 
Equal proportions of IC/BPS and OAB patients endorsed urgency symptoms with varying definitions ("strong need to urinate with little or no warning," "difficulty postponing urination," "strong urge or pressure that signaled the need to urinate immediately"), and varying timeframes (7 days, 1 month, 3 months). Similar proportions of both groups ( $60 \%)$ also reported that the urgency occurred "suddenly" instead of more gradually over a period of minutes or hours.

All subjects were asked about the use of antimuscarinic agents ("In the last 3 months have you been taking medications for urinary incontinence and/or urgency, such as Detrol or Ditropan?"). Use of these medications was reported by 34 IC patients (18\%) and $43 \mathrm{OAB}$ patients (51\%). Subgroup analysis based on the use of these medications was performed, but the overall study findings were not affected in any meaningful ways (data not shown).

\section{DISCUSSION}

These results indicate that current urinary urgency was a commonly reported symptom by women diagnosed with IC/BPS or OAB, and that there was considerable overlap in how these urgency symptoms are described by women with each condition. This suggests that "urgency" is not a well-defined and commonly understood symptom, and that it cannot be utilized by itself to discriminate between IC/BPS and OAB. This finding is consistent with recent consensus panel definitions for IC/BPS, which do not include urgency as a criterion for diagnosis. ${ }^{10-12}$ The use of the term "sudden" is included in the International Continence Society (ICS) definition of urgency, ${ }^{13}$ and is perhaps designed to separate OAB from IC/BPS. However, our data suggest that "sudden" onset of urgency appears to be a common characteristic of both conditions.

Although no set of responses was unique to one patient group, some differences were observed. IC/BPS patients primarily reported urgency due to pain, pressure, or discomfort, while $\mathrm{OAB}$ patients more commonly indicated urgency due to fear of leakage. Compared with IC/BPS, urgency in OAB more often resulted in urinary incontinence, and was perceived to be more of a problem. Only $11 \%$ of IC/BPS patients reported urgency due to a fear of leakage, and $13.4 \%$ report leaking urine "right away" once the feeling of urgency starts. It is possible that these patients may represent late-stage IC/BPS patients with fibrotic or contracted bladders, or perhaps these were women with both conditions but the OAB was not recognized by physicians. Unfortunately, we lack additional clinical information to investigate these questions further. These trends are consistent with clinical descriptions and formal definitions that have been developed for these conditions, ${ }^{1,2}$ but the correlation between these symptoms and their expected clinical diagnoses was relatively poor. For instance, over $40 \%$ of women diagnosed with $\mathrm{OAB}$ reported urgency due to pain, pressure, or discomfort rather than due to a fear of incontinence. Furthermore, $49 \%$ of IC/BPS patients report that the urgency would cause them to leak if they have to wait a while before using the bathroom. However, we do not know how many of these women actually experienced leakage.

A critical question of this analysis relates to the selection criteria for the IC/BPS and OAB cohorts. These cohorts were identified based on clinical diagnoses assigned by experienced clinicians. Each referring physician was asked to recruit patients with diagnoses of IC/BPS or OAB who were currently symptomatic. It is acknowledged that we do not have data about the exact diagnostic tests used to make the diagnoses, or other potentially relevant clinical conditions (e.g., pelvic floor dysfunction). In fact, our results would suggest that some of the selection criteria may have been faulty. For instance, it is difficult to explain the $14 \%$ of IC/BPS patients who report leakage of urine "right away" once the feeling of urgency starts. The use of more rigorous inclusion criteria may have yielded more discriminatory results. However, any such criteria are likely to have their own limitations. For instance, the NIDDK criteria for IC/BPS allow the presence of either pain or urgency, and the typical cystoscopic findings of IC/BPS are nonspecific. ${ }^{14,15}$ The underlying problem is that neither IC/BPS nor OAB has an objective and reliable symptom or test to confirm the diagnosis. In practice, the diagnosis is based on clinical judgment and exclusion of other specific conditions that could explain the symptoms and, in some case, the success or failure of trials of medications.

Additional limitations deserve mention. Since the clinical diagnoses occurred prior to our symptom assessment, it is possible that the nature of the symptoms or treatments changed with time. We do not have data about the time interval between the diagnosis (or symptom onset) and the interview, and duration of disease may potentially have an impact on symptoms. In addition, the limited set of questions employed in this analysis may have missed an important aspect of the symptom experience of urgency. For instance, it is possible that OAB symptoms are experienced by many patients as "pressure," but not "pain." If these specific descriptors had been separated, our findings may have been more discriminative. Finally, the study was limited to women, as the design of the parent RICE study was to investigate IC/PBS symptoms in women only. Despite these limitations, we feel that this systematic comparison of urgency symptoms in IC/BPS and OAB does provide valuable information about the complexity of these symptoms.

\section{CONCLUSIONS}

The symptom of urgency was commonly reported in some form by women who had been diagnosed with IC/BPS or OAB. In IC/BPS patients, the urgency was predominantly due to pain, pressure, or discomfort, while in $\mathrm{OAB}$ patients, the urgency more commonly due to a fear of incontinence. However, there was considerable overlap in these and other urgency symptom descriptors across the two groups. These findings reinforce the clinical observation that it can be challenging to differentiate between these two conditions.

\section{REFERENCES}

1. Abrams P, Cardozo L, Fall M, et al. The standardization of terminology of lower urinary tract function: report from the Standardisation Sub-committee of the International Continence Society. Neurourol Urodyn 2002;21:167-78.

2. Abrams $P$, Hanno $P$, Wein A. Overactive bladder and painful bladder syndrome: there need not be confusion. Neurourol Urodyn 2005;24:149-50.

3. Hanno PM. Re-imagining interstitial cystitis. Urol Clin N Am 2008;35:91-9.

4. Parsons CL, Dell J, Stanford EJ, et al. Increased prevalence of interstitial cystitis: previously unrecognized urologic and gynecologic cases identified using a new symptom questionnaire and intravesical potassium sensitivity. Urology 2002;60:573-8.

5. Keller ML, McCarthy DO, Neider RS. Measurement of symptoms of interstitial cystitis. Urol Clin N Am 1994;21:67-71.

6. O'Leary MP, Sant GR, Fowler FJ, JR., et al. The interstitial cystitis symptom index and problem index. Urology 1997;49(Suppl 5A):58-63.

7. Coyne K, Revicki D, Hunt T, et al. Psychometric validation of an overactive bladder symptom and health-related quality of life questionnaire: the OAB-q. OOL Res 2002;11:563-74.

8. Diggs C, Meyer WA, Langenberg P, et al. Assessing urgency in interstitial cystitis/painful bladder syndrome. Urology 2007;69:210-4. 
9. Berry SH, Bogart LM, Pham C, et al. Development, validation and testing of an epidemiologic case definition for interstitial cystitis/painful bladder syndrome. J Urol 2010;183:1848-52.

10. Hanno P, Dmochowski RR. Status of international consensus on interstitial cystitis/bladder pain syndrome/painful bladder syndrome: 2008 snapshot Neurourol Urodyn 2009;28:274-86

11. Van De Merwe J, Nordling J, Bouchelouche K, et al. Diagnostic criteria, classification, and nomenclature for painful bladder syndrome/interstitial cystitis: an ESSIC proposal. Eur Urol 2008;53:60-7.

12. Hanno P, Lin A, Nordling J, et al. Bladder pain syndrome. Neurourol Urodyn 2010;29:191-8
13. Abrams P, Cardozo L, Fall $M$, et al. The standardization of terminology of lower urinary tract function: report from the Standardization Subcommittee of the International Continence Society. Neurourol Urodyn 2002;21: 167-78.

14. Waxman JA, Sulak PJ, Kuehl TJ. Cystoscopic findings consistent with interstitial cystitis in normal women undergoing tubal ligation. J Urol 1998;160: 1663-7.

15. Furuya R, Masumori N, Furuya S, et al. Glomerulations observed during transurethral resection of the prostate for patients with lower urinary tract symptoms suggestive of benign prostatic hyperplasia is a common finding but no predictor of clinical outcome. Urology 2007;70:922-6. 